International Journal of Pure and Applied Mathematics

Volume 87 No. 3 2013, 355-369

ISSN: 1311-8080 (printed version); ISSN: 1314-3395 (on-line version)

url: http://www.ijpam.eu

doi: http://dx.doi.org/10.12732/ijpam.v87i3.1

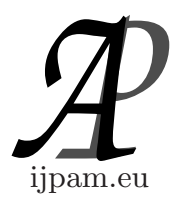

\title{
ON THE OSTROWSKI-GRÜSS-LIKE TYPE INEQUALITY AND AN ANALOGUE OF OSTROWSKI-LIKE TYPE INEQUALITY
}

\author{
Jaekeun Park \\ Department of Mathematics \\ Hanseo University \\ Seosan, Chungnam, 356-706, KOREA
}

\begin{abstract}
In this article, we establish some new generalizations of the Ostrowski-Grüss-like type inequality for twice continuously differentiable functions and new generalized analogues of the Ostrowski-like type inequality in three different cases.
\end{abstract}

AMS Subject Classification: 26A24, 26A51, 26B25

Key Words: Ostrowski's inequality, Grüss' inequality

\section{Introduction}

In [10], Ostrowski proved the following theorem:

Theorem 1.1. Let $f: I \rightarrow R$ be a differentiable mapping in the interior $I^{0}$ of an interval $I$ and $a, b \in I^{0}$ with $a<b$. If $\left|f^{\prime}(t)\right| \leq M$ for any $t \in[a, b]$, then we have

$$
\left|f(x)-\frac{1}{b-a} \int_{a}^{b} f(t) d t\right| \leq\left[\frac{1}{4}+\frac{\left(x-\frac{a+b}{2}\right)^{2}}{(b-a)^{2}}\right](b-a) M
$$

for $x \in[a, b]$.

Received: February 18, 2013

(c) 2013 Academic Publications, Ltd. url: www.acadpubl.eu 
Recently there has been considerable interest in the study of Ostrowski type inequalities. Some new types of inequalities are established, for example inequalities of Ostrowski-Grüss type and inequalities of Ostrowski-Chebyshev type. In [8], Milovanović and Pečarić gave a generalization of Ostrowski's inequality and some related applications.

An Ostrowski-Grüss type inequality was given for the first time by Dragomir and Wang in [3]. In [5], Matić et al. generalized and improved this inequality. For generalizations, improvements and recent results, see the papers $[3,4,11$, $12,13,14]$. Recently, in [13], Ujević proved the following theorem:

Theorem 1.2. Let $f: I \rightarrow R$ be a differentiable function in the interior $I^{0}$ of an interval $I$ in $R$ and $a, b \in I^{0}$ with $a<b$. If there exist constants $\gamma, \Gamma \in R$ such that $\gamma \leq f^{\prime}(t) \leq \Gamma$ for any $t \in[a, b]$ and $f^{\prime} \in L^{1}[a, b]$, then we have

$$
\begin{aligned}
& \left|f(x)-\left(x-\frac{a+b}{2}\right) \frac{f(b)-f(a)}{b-a}-\frac{1}{b-a} \int_{a}^{b} f(t) d t\right| \\
& \leq \frac{(b-a)}{2}(S-\gamma)
\end{aligned}
$$

and

$$
\begin{aligned}
& \left|f(x)-\left(x-\frac{a+b}{2}\right) \frac{f(b)-f(a)}{b-a}-\frac{1}{b-a} \int_{a}^{b} f(t) d t\right| \\
& \leq \frac{(b-a)}{2}(\Gamma-S)
\end{aligned}
$$

where $S=\frac{f(b)-f(a)}{b-a}$.

Theorem 1.3. Let $f: I \rightarrow R$ be a twice continuously differentiable function in the interior $I^{0}$ of an interval $I$ in $R$ with $f^{\prime \prime} \in L^{2}[a, b]$ and $a, b \in I^{0}$ with $a<b$. Then we have

$$
\begin{aligned}
& \left|f(x)-\left(x-\frac{a+b}{2}\right) \frac{f(b)-f(a)}{b-a}-\frac{1}{b-a} \int_{a}^{b} f(t) d t\right| \\
& \leq \frac{(b-a)^{\frac{3}{2}}}{2 \sqrt{3} \pi}\left\|f^{\prime \prime}\right\|_{2},
\end{aligned}
$$

for $x \in[a, b]$.

For two absolutely continuous functions $f, g:[a, b] \rightarrow R$ such that $f, g, f g \in$ $L^{1}[a, b]$, the Chebyshev functional $T(\cdot, \cdot)$ is defined by

$$
T_{a}^{b}(f, g)=\frac{1}{(b-a)} \int_{a}^{b} f(x) g(x) d x-\frac{1}{(b-a)^{2}} \int_{a}^{b} f(x) d x \int_{a}^{b} g(x) d x .
$$


A well-known inequality in the literature, which is related to the Chebyshev functional, is the Ostrowski inequality [8].

The Ostrowski type inequality also plays an important role in numerical quadrature rules [7].

For various generalizations, extensions and related Ostrowski type inequalities for functions of one or several variables, see the monograph [2] and the references therein. For the related result, see the papers $[1,2,5,8,7]$.

In [6], Masjed-Jamei and Dragomir introduced a new analogue of the Ostrowski type inequality in three different cases and apply them for some quadrature rules and proved the following theorem:

Theorem 1.4. Let $f: I \rightarrow R$ be a differentiable function in the interior $I^{0}$ of an interval $I$ in $R$ and $a, b \in I^{0}$ with $a<b$. If $\alpha(x) \leq f^{\prime}(x) \leq \beta(x)$ for any $\alpha, \beta \in C[a, b]$ and $x \in[a, b]$, then the following inequality holds:

$$
\begin{aligned}
& \frac{1}{b-a}\left\{\int_{a}^{x}(t-a) \alpha(t) d t+\int_{x}^{b}(t-b) \beta(t) d t\right\} \\
& \leq f(x)-\frac{1}{b-a} \int_{a}^{b} f(t) d t \\
& \leq \frac{1}{b-a}\left\{\int_{a}^{x}(t-a) \beta(t) d t+\int_{x}^{b}(t-b) \alpha(t) d t\right\} .
\end{aligned}
$$

Theorem 1.5. Let $f: I \rightarrow R$ be a differentiable function in the interior $I^{0}$ of an interval $I$ in $R$ and $a, b \in I^{0}$ with $a<b$. If $\alpha(x) \leq f^{\prime}(x)$ for any $\alpha \in C[a, b]$ and $x \in[a, b]$, then the following inequalities hold:

$$
\begin{aligned}
& \frac{1}{b-a}\left[\int_{a}^{x}(t-a) \alpha(t) d t+\int_{x}^{b}(t-b) \alpha(t) d t\right. \\
& \quad-\max \{x-a, b-x\}\left\{f(b)-f(a)-\int_{a}^{b} \alpha(t) d t\right\} \\
& \leq f(x)-\frac{1}{b-a} \int_{a}^{b} f(t) d t \\
& \leq \frac{1}{b-a}\left[\int_{a}^{x}(t-a) \alpha(t) d t+\int_{x}^{b}(t-b) \alpha(t) d t\right. \\
& \quad+\max \{x-a, b-x\}\left\{f(b)-f(a)-\int_{a}^{b} \alpha(t) d t\right\} .
\end{aligned}
$$


In this article, in Section 2 we will prove the Ostrowski-Grüss-like type inequalities similar to above Theorem 1.2 and Theorem 1.3 but now involving twice differentiable functions, and in Section 3 we will introduce a new generalized analogue of the Ostrowski-like type inequality in three different cases by using the more generalized kernel function.

\section{Ostrowski-Grüss Type Inequality for Twice Differentiable Functions}

In this section we will give the generalization of the Ostrowski-Grüss-like type inequality for twice continuously differentiable functions. To do this, let us consider the following kernel function $K_{1}(\cdot, \cdot):\left[a+\lambda \frac{b-a}{2}, b-\lambda \frac{b-a}{2}\right]^{2} \rightarrow R$ defined by

$$
K_{1}(x, t)= \begin{cases}\frac{t}{n}\left\{t-n\left(a+\lambda \frac{b-a}{2}\right)\right\} & t \in\left[a+\lambda \frac{b-a}{2}, x\right] \\ \frac{t}{n}\left\{t-n\left(b-\lambda \frac{b-a}{2}\right)\right\} & t \in\left[x, b-\lambda \frac{b-a}{2}\right]\end{cases}
$$

for any $\lambda \in[0,1]$ and integer $n \geq 2$.

To begin with, let us start with the following theorem.

Theorem 2.1. Let $f: I \rightarrow R$ be a twice continuously differentiable function in the interior $I^{0}$ of an interval $I$ in $R$ and $a, b \in I^{0}$ with $a<b$. If there exist constants $\gamma, \Gamma \in R$ such that $\gamma \leq f^{\prime \prime}(t) \leq \Gamma$ for any $t \in\left[a+\lambda \frac{b-a}{2}, b-\lambda \frac{b-a}{2}\right]$ for $\lambda \in[0,1]$ and $f^{\prime \prime} \in L^{2}\left[a+\lambda \frac{b-a}{2}, b-\lambda \frac{b-a}{2}\right]$, then, for any integer $n \geq 2$ we have the following inequalities:

$$
\begin{gathered}
\left|R_{n}(x)\right| \leq \frac{(1-\lambda)(b-a)}{12 n}[\{3 n(\lambda-2)+2(\lambda+2)\} a \\
+\{8-(3 n+2) \lambda\} b](S(\lambda)-\gamma)
\end{gathered}
$$

and

$$
\begin{gathered}
\left|R_{n}(x)\right| \leq \frac{(1-\lambda)(b-a)}{12 n}[\{3 n(\lambda-2)+2(\lambda+2)\} a \\
+\{8-(3 n+2) \lambda\} b](\Gamma-S(\lambda)),
\end{gathered}
$$

where

$$
R_{n}(x)
$$




$$
\begin{aligned}
= & x f^{\prime}(x)-f(x)+\left(\frac{2}{n(1-\lambda)(b-a)}\right) \int_{a+\lambda \frac{\mathrm{b}-\mathrm{a}}{2}}^{b-\lambda \frac{\mathrm{b}-\mathrm{a}}{2}} f(t) d t \\
+ & \left(\frac{n-1}{n}\right)\left\{\frac{\left(a+\lambda \frac{b-a}{2}\right)^{2} f^{\prime}\left(a+\lambda \frac{b-a}{2}\right)-\left(b-\lambda \frac{b-a}{2}\right)^{2} f^{\prime}\left(b-\lambda \frac{b-a}{2}\right)}{(1-\lambda)(b-a)}\right\} \\
- & \left(\frac{n-2}{n}\right)\left\{\frac{\left(a+\lambda \frac{b-a}{2}\right) f\left(a+\lambda \frac{b-a}{2}\right)-\left(b-\lambda \frac{b-a}{2}\right) f\left(b-\lambda \frac{b-a}{2}\right)}{(1-\lambda)(b-a)}\right\} \\
- & \left\{\frac{1}{2} x^{2}-\left(\frac{3 n-2}{24 n}\right)\left\{(1-\lambda)^{2}(b-a)^{2}+3(a+b)^{2}\right\}\right\} \\
& \times\left\{\frac{f^{\prime}\left(b-\lambda \frac{b-a}{2}\right)-f^{\prime}\left(a+\lambda \frac{b-a}{2}\right)}{(1-\lambda)(b-a)}\right\},
\end{aligned}
$$

and

$$
S(\lambda)=\frac{f^{\prime}\left(b-\lambda \frac{b-a}{2}\right)-f^{\prime}\left(a+\lambda \frac{b-a}{2}\right)}{(1-\lambda)(b-a)}
$$

Proof. By the definition of $K_{1}(x, t)$, we have

$$
\begin{aligned}
\int_{a+\lambda \frac{\mathrm{b}-\mathrm{a}}{2}}^{b-\lambda \frac{\mathrm{b}-\mathrm{a}}{2}} K_{1}(x, t) f^{\prime \prime}(t) d t \\
=\frac{2}{n} \int_{a+\lambda \frac{\mathrm{b}-\mathrm{a}}{2}}^{b-\lambda \frac{\mathrm{b}-\mathrm{a}}{2}} f(t) d t \\
\quad+(1-\lambda)(b-a) x f^{\prime}(x)-(1-\lambda)(b-a) f(x) \\
\quad+\left(\frac{n-1}{n}\right)\left\{\left(a+\lambda \frac{b-a}{2}\right)^{2} f^{\prime}\left(a+\lambda \frac{b-a}{2}\right)\right. \\
\left.\quad-\left(b-\lambda \frac{b-a}{2}\right)^{2} f^{\prime}\left(a+\lambda \frac{b-a}{2}\right)\right\} \\
\quad-\left(\frac{n-2}{n}\right)\left\{\left(a+\lambda \frac{b-a}{2}\right) f\left(a+\lambda \frac{b-a}{2}\right)\right. \\
\left.\quad-\left(b-\lambda \frac{b-a}{2}\right) f\left(b-\lambda \frac{b-a}{2}\right)\right\} .
\end{aligned}
$$

By the simple calculation, we have

$$
\frac{1}{(1-\lambda)(b-a)} \int_{a+\lambda \frac{\mathrm{b}-\mathrm{a}}{2}}^{b-\lambda \frac{\mathrm{b}-\mathrm{a}}{2}} K_{1}(x, t) d t
$$




$$
=\left\{\frac{1}{2} x^{2}-\left(\frac{3 n-2}{24 n}\right)\left((1-\lambda)^{2}(b-a)^{2}+3(a+b)^{2}\right)\right\}
$$

and

$$
\int_{a+\lambda \frac{\mathrm{b}-\mathrm{a}}{2}}^{b-\lambda \frac{\mathrm{b}-\mathrm{a}}{2}} f^{\prime \prime}(t) d t=f^{\prime}\left(b-\lambda \frac{b-a}{2}\right)-f^{\prime}\left(a+\lambda \frac{b-a}{2}\right) .
$$

By using (10),(11) and (12), we get

$$
\begin{aligned}
& T_{a+\lambda \frac{\mathrm{b}-\mathrm{a}}{2}}^{b-\lambda \frac{\mathrm{b}-\mathrm{a}}{2}}\left(K_{1}(x, \cdot), f^{\prime \prime}\right) \\
&=\left(\frac{2}{n(1-\lambda)(b-a)}\right) \int_{a+\lambda \frac{\mathrm{b}-\mathrm{a}}{2}}^{b-\lambda \frac{\mathrm{b}-\mathrm{a}}{2}} f(t) d t+x f^{\prime}(x)-f(x) \\
&+\left(\frac{n-1}{n}\right)\left\{\frac{\left(a+\lambda \frac{b-a}{2}\right)^{2} f^{\prime}\left(a+\lambda \frac{b-a}{2}\right)-\left(b-\lambda \frac{b-a}{2}\right)^{2} f^{\prime}\left(a+\lambda \frac{b-a}{2}\right)}{(1-\lambda)(b-a)}\right\} \\
&-\left(\frac{n-2}{n}\right)\left\{\frac{\left(a+\lambda \frac{b-a}{2}\right) f\left(a+\lambda \frac{b-a}{2}\right)-\left(b-\lambda \frac{b-a}{2}\right) f\left(b-\lambda \frac{b-a}{2}\right)}{(1-\lambda)(b-a)}\right\} \\
&-\left\{\frac{1}{2} x^{2}-\left(\frac{3 n-2}{24 n}\right)\left((1-\lambda)^{2}(b-a)^{2}+3(a+b)^{2}\right)\right\} \\
& \times\left\{\frac{f^{\prime}\left(b-\lambda \frac{b-a}{2}\right)-f^{\prime}\left(a+\lambda \frac{b-a}{2}\right)}{(1-\lambda)(b-a)}\right\} .
\end{aligned}
$$

Here, let

$$
R_{n}(x)=T_{a+\lambda \frac{\mathrm{b}-\mathrm{a}}{2}}^{b-\lambda \frac{\mathrm{b}-\mathrm{a}}{2}}\left(K_{1}(x, \cdot), f^{\prime \prime}\right) .
$$

By the definition of Chebyshev functional, we have

$$
\begin{aligned}
R_{n}(x)= & \frac{1}{(1-\lambda)(b-a)} \int_{a+\lambda \frac{\mathrm{b}-\mathrm{a}}{2}}^{b-\lambda \frac{\mathrm{b}-\mathrm{a}}{2}} K_{1}(x, t) f^{\prime \prime}(t) d t \\
& -\frac{1}{(1-\lambda)^{2}(b-a)^{2}} \int_{a+\lambda \frac{\mathrm{b}-\mathrm{a}}{2}}^{b-\lambda \frac{\mathrm{b}-\mathrm{a}}{2}} f^{\prime \prime}(t) d t \int_{a+\lambda \frac{\mathrm{b}-\mathrm{a}}{2}}^{b-\lambda \frac{\mathrm{b}-\mathrm{a}}{2}} K_{1}(x, t) d t \\
= & \frac{1}{(1-\lambda)(b-a)} \int_{a+\lambda \frac{\mathrm{b}-\mathrm{a}}{2}}^{b-\lambda \frac{\mathrm{b}-\mathrm{a}}{2}}\left(f^{\prime \prime}(t)-C\right) \\
& \times\left[K_{1}(x, t)-\frac{1}{(1-\lambda)(b-a)} \int_{a+\lambda \frac{\mathrm{b}-\mathrm{a}}{2}}^{b-\lambda \frac{\mathrm{b}-\mathrm{a}}{2}} K_{1}(x, s) d s\right] d t .
\end{aligned}
$$


Also, we know that

$$
\int_{a+\lambda \frac{\mathrm{b}-\mathrm{a}}{2}}^{b-\lambda \frac{\mathrm{b}-\mathrm{a}}{2}}\left[K_{1}(x, t)-\frac{1}{(1-\lambda)(b-a)} \int_{a+\lambda \frac{\mathrm{b}-\mathrm{a}}{2}}^{b-\lambda \frac{\mathrm{b}-\mathrm{a}}{2}} K_{1}(x, s) d s\right] d t=0 .
$$

So, if we choose $C=\gamma$, then we get

$$
\begin{aligned}
R_{n}(x)= & \frac{1}{(1-\lambda)(b-a)} \int_{a+\lambda \frac{\mathrm{b}-\mathrm{a}}{2}}^{b-\lambda \frac{\mathrm{b}-\mathrm{a}}{2}}\left(f^{\prime \prime}(t)-\gamma\right) \\
& \quad \times\left[K_{1}(x, t)-\frac{1}{(1-\lambda)(b-a)} \int_{a+\lambda \frac{\mathrm{b}-\mathrm{a}}{2}}^{b-\lambda \frac{\mathrm{b}-\mathrm{a}}{2}} K_{1}(x, s) d s\right] d t .
\end{aligned}
$$

Note that

$$
\begin{aligned}
& \int_{a+\lambda \frac{\mathrm{b}-\mathrm{a}}{2}}^{b-\lambda \frac{\mathrm{b}-\mathrm{a}}{2}}\left|\left(f^{\prime \prime}(t)-\gamma\right)\right| d t \\
& =f^{\prime}\left(b-\lambda \frac{b-a}{2}\right)-f^{\prime}\left(a+\lambda \frac{b-a}{2}\right)-\gamma(1-\lambda)(b-a) \\
& =(1-\lambda)(b-a)(S(\lambda)-\gamma)
\end{aligned}
$$

where

$$
S(\lambda)=\frac{f^{\prime}\left(b-\lambda \frac{b-a}{2}\right)-f^{\prime}\left(a+\lambda \frac{b-a}{2}\right)}{(1-\lambda)(b-a)}
$$

Also, we have

$$
\begin{aligned}
\left|R_{n}(x)\right| \leq & \frac{1}{(1-\lambda)(b-a)} \int_{a+\lambda \frac{\mathrm{b}-\mathrm{a}}{2}}^{b-\lambda \frac{\mathrm{b}-\mathrm{a}}{2}}\left|\left(f^{\prime \prime}(t)-\gamma\right)\right| d t \\
& \times \max \left|K_{1}(x, t)-\frac{1}{(1-\lambda)(b-a)} \int_{a+\lambda \frac{\mathrm{b}-\mathrm{a}}{2}}^{b-\lambda \frac{\mathrm{b}-\mathrm{a}}{2}} K_{1}(x, s) d s\right| .
\end{aligned}
$$

Note that

$$
\begin{aligned}
& \max \left|K_{1}(x, t)-\frac{1}{(1-\lambda)(b-a)} \int_{a+\lambda \frac{\mathrm{b}-\mathrm{a}}{2}}^{b-\lambda \frac{\mathrm{b}-\mathrm{a}}{2}} K_{1}(x, s) d s\right| \\
& =\max \left|K_{1}(x, t)-\left\{\frac{1}{2} x^{2}-\left(\frac{3 n-2}{24 n}\right)\left((1-\lambda)^{2}(b-a)^{2}+3(a+b)^{2}\right)\right\}\right| \\
& =\frac{(1-\lambda)(b-a)}{12 n}[\{3 n(\lambda-2)+2(\lambda+2)\} a+\{8-(2+3 n) \lambda\} b] .
\end{aligned}
$$


By (15) and (16), we get the desired result (8):

$$
\begin{aligned}
& \left|R_{n}(x)\right| \\
& \leq(1-\lambda)(b-a)(S(\lambda)-\gamma) \\
& \quad \times\left(\frac{1}{12 n}\right)[\{3 n(\lambda-2)+2(\lambda+2)\} a+\{8-(2+3 n) \lambda\} b] \\
& =\frac{(1-\lambda)(b-a)}{12 n}[\{3 n(\lambda-2)+2(\lambda+2)\} a \\
& \quad+\{8-(2+3 n) \lambda\} b](S(\lambda)-\gamma),
\end{aligned}
$$

Secondly, if we choose $C=\Gamma$ in (13), then by a similar argument we get

$$
\begin{aligned}
\left|R_{n}(x)\right|= & \frac{1}{(1-\lambda)(b-a)} \int_{a+\lambda \frac{\mathrm{b}-\mathrm{a}}{2}}^{b-\lambda \frac{\mathrm{b}-\mathrm{a}}{2}}\left|\left(f^{\prime \prime}(t)-\Gamma\right)\right| d t \\
& \times \max \left|K_{1}(x, t)-\frac{1}{(1-\lambda)(b-a)} \int_{a+\lambda \frac{\mathrm{b}-\mathrm{a}}{2}}^{b-\lambda \frac{\mathrm{b}-\mathrm{a}}{2}} K_{1}(x, s) d s\right|
\end{aligned}
$$

and

$$
\begin{aligned}
& \int_{a+\lambda \frac{\mathrm{b}-\mathrm{a}}{2}}^{b-\lambda \frac{\mathrm{b}-\mathrm{a}}{2}}\left|\left(f^{\prime \prime}(t)-\Gamma\right)\right| d t \\
& =-\left\{f^{\prime}\left(b-\lambda \frac{b-a}{2}\right)-f^{\prime}\left(a+\lambda \frac{b-a}{2}\right)\right\}+\Gamma(1-\lambda)(b-a) \\
& =(1-\lambda)(b-a)(\Gamma-S(\lambda)) .
\end{aligned}
$$

By (16),(17) and (18), we get the desired result (9).

Theorem 2.2. Let $f: I \rightarrow R$ be a twice continuously differentiable function in the interior $I^{0}$ of an interval $I$ in $R$ and $a, b \in I^{0}$ with $a<b$. If $f^{\prime \prime} \in L_{2}\left[a+\lambda \frac{b-a}{2}, b-\lambda \frac{b-a}{2}\right]$ for $\lambda \in[0,1]$, then, for any integer $n \geq 2$ we have the following inequalities:

$$
\begin{array}{r}
\left|R_{n}(x)\right| \leq \frac{(1-\lambda)(b-a)}{12 n}[\{3 n(\lambda-2)+2(\lambda+2)\} a \\
+\{8-(3 n+2) \lambda\} b]\left(S(\lambda)-f^{\prime \prime}\left(\frac{a+b}{2}\right)\right)
\end{array}
$$

where

$$
S(\lambda)=\frac{f^{\prime}\left(b-\lambda \frac{b-a}{2}\right)-f^{\prime}\left(a+\lambda \frac{b-a}{2}\right)}{(1-\lambda)(b-a)}
$$


Proof. Let $R_{n}(x)$ be defined as in the equality (13) with $C \in R$ an arbitrary constant. If we choose $C=f^{\prime \prime}\left(\frac{a+b}{2}\right)$, we get

$$
\begin{aligned}
& \left|R_{n}(x)\right| \\
& \leq \frac{1}{(1-\lambda)(b-a)} \int_{a+\lambda \frac{\mathrm{b}-\mathrm{a}}{2}}^{b-\lambda \frac{\mathrm{b}-\mathrm{a}}{2}}\left|f^{\prime \prime}(t)-f^{\prime \prime}\left(\frac{a+b}{2}\right)\right| d t \\
& \quad \times \max \left|K_{1}(x, t)-\left\{\frac{1}{2} x^{2}-\left(\frac{3 n-2}{24 n}\right)\left\{(1-\lambda)^{2}(b-a)^{2}+3(a+b)^{2}\right\}\right\}\right| \\
& =\frac{(1-\lambda)(b-a)}{12 n}[\{3 n(\lambda-2)+2(\lambda+2)\} a+\{8-(2+3 n) \lambda\} b] \\
& \quad \times\left(S(\lambda)-f^{\prime \prime}\left(\frac{a+b}{2}\right)\right),
\end{aligned}
$$

which completes the proof.

\section{Ostrowski-Type Inequality for Differentiable Functions}

In this section we will prove new analogues of the Ostrowski-like type inequality for differentiable functions in three different cases, which are generalizations of Theorem 3.1 and Theorem 3.2.

To do this, let us consider the following kernel function $K_{2}(\cdot, \cdot)$ : $[a+$ $\left.\lambda \frac{b-a}{2}, b-\lambda \frac{b-a}{2}\right]^{2} \rightarrow R$ defined by

$$
K_{2}(x, t)= \begin{cases}t-\left(a+\lambda \frac{b-a}{2}\right) & t \in\left[a+\lambda \frac{b-a}{2}, x\right] \\ t-\left(b-\lambda \frac{b-a}{2}\right) & t \in\left[x, b-\lambda \frac{b-a}{2}\right]\end{cases}
$$

for any $\lambda \in[0,1]$.

Theorem 3.1. Let $f: I \rightarrow R$ be a differentiable function in the interior $I^{0}$ of an interval $I$ in $R$ and $a, b \in I^{0}$ with $a<b$. If $\alpha(x) \leq f^{\prime}(x) \leq \beta(x)$ for any $\alpha, \beta \in C\left[a+\lambda \frac{b-a}{2}, b-\lambda \frac{b-a}{2}\right]$ and $x \in\left[a+\lambda \frac{b-a}{2}, b-\lambda \frac{b-a}{2}\right]$ for $\lambda \in[0,1]$, then the following inequality holds:

$$
\begin{aligned}
\int_{a+\lambda \frac{\mathrm{b}-\mathrm{a}}{2}}^{x} & \left\{t-\left(a+\lambda \frac{b-a}{2}\right)\right\} \alpha(t) d t \\
+ & \int_{x}^{b-\lambda \frac{\mathrm{b}-\mathrm{a}}{2}}\left\{t-\left(b-\lambda \frac{b-a}{2}\right)\right\} \beta(t) d t
\end{aligned}
$$




$$
\begin{aligned}
& \leq(1-\lambda)(b-a) f(x)-\int_{a+\lambda \frac{\mathrm{b}-\mathrm{a}}{2}}^{b-\lambda \frac{\mathrm{b}-\mathrm{a}}{2}} f(t) d t \\
& \leq \int_{a+\lambda \frac{\mathrm{b}-\mathrm{a}}{2}}^{x}\left\{t-\left(a+\lambda \frac{b-a}{2}\right)\right\} \beta(t) d t \\
& \quad+\int_{x}^{b-\lambda \frac{\mathrm{b}-\mathrm{a}}{2}}\left\{t-\left(b-\lambda \frac{b-a}{2}\right)\right\} \alpha(t) d t .
\end{aligned}
$$

Proof. By the definition $K_{2}(\cdot, \cdot)$, we have

$$
\int_{a+\lambda \frac{\mathrm{b}-\mathrm{a}}{2}}^{b-\lambda \frac{\mathrm{b}-\mathrm{a}}{2}} K_{2}(x, t) f^{\prime}(t) d t=(1-\lambda)(b-a) f(x)-\int_{a+\lambda \frac{\mathrm{b}-\mathrm{a}}{2}}^{b-\lambda \frac{\mathrm{b}-\mathrm{a}}{2}} f(t) d t .
$$

By referring to the kernel $K_{2}(\cdot, \cdot)$ and the identity $(21)$ we have

$$
\begin{aligned}
& \int_{a+\lambda \frac{\mathrm{b}-\mathrm{a}}{2}}^{b-\lambda \frac{\mathrm{b}-\mathrm{a}}{2}} K_{2}(x, t)\left\{f^{\prime}(t)-\frac{\alpha(t)+\beta(t)}{2}\right\} d t \\
& =(1-\lambda)(b-a) f(x)-\int_{a+\lambda \frac{\mathrm{b}-\mathrm{a}}{2}}^{b-\lambda \frac{\mathrm{b}-\mathrm{a}}{2}} f(t) d t \\
& -\frac{1}{2}\left[\int_{a+\lambda \frac{\mathrm{b}-\mathrm{a}}{2}}^{x}\left\{t-\left(a+\lambda \frac{b-a}{2}\right)\right\}\{\alpha(t)+\beta(t)\} d t\right. \\
& \left.+\int_{x}^{b-\lambda \frac{\mathrm{b}-\mathrm{a}}{2}}\left\{t-\left(b-\lambda \frac{b-a}{2}\right)\right\}\{\alpha(t)+\beta(t)\} d t\right] .
\end{aligned}
$$

On the other hand, by the fact that $\alpha(x) \leq f^{\prime}(x) \leq \beta(x)$ for any $\alpha, \beta \in$ $C\left[a+\lambda \frac{b-a}{2}, b-\lambda \frac{b-a}{2}\right]$ and for $\lambda \in[0,1]$, we have

$$
\left|f^{\prime}(t)-\frac{\alpha(t)+\beta(t)}{2}\right| \leq\left|\beta(t)-\frac{\alpha(t)+\beta(t)}{2}\right|=\frac{\beta(t)-\alpha(t)}{2} .
$$

Therefore, by (22) and (23) one can conclude that

$$
\begin{aligned}
& \mid(1-\lambda)(b-a) f(x)-\int_{a+\lambda \frac{\mathrm{b}-\mathrm{a}}{2}}^{b-\lambda \frac{\mathrm{b}-\mathrm{a}}{2}} f(t) d t \\
& \quad-\frac{1}{2}\left[\int_{a+\lambda \frac{\mathrm{b}-\mathrm{a}}{2}}^{x}\left\{t-\left(a+\lambda \frac{b-a}{2}\right)\right\}\{\alpha(t)+\beta(t)\} d t\right. \\
& \left.\quad+\int_{x}^{b-\lambda \frac{\mathrm{b}-\mathrm{a}}{2}}\left\{t-\left(b-\lambda \frac{b-a}{2}\right)\right\}\{\alpha(t)+\beta(t)\} d t\right] \mid
\end{aligned}
$$




$$
\begin{aligned}
\leq & \int_{a+\lambda \frac{\mathrm{b}-\mathrm{a}}{2}}^{b-\lambda \frac{\mathrm{b}-\mathrm{a}}{2}}\left|K_{2}(x, t)\right|\left|f^{\prime}(t)-\frac{\alpha(t)+\beta(t)}{2}\right| d t \\
\leq & \int_{a+\lambda \frac{\mathrm{b}-\mathrm{a}}{2}}^{b-\lambda \frac{\mathrm{b}-\mathrm{a}}{2}}\left|K_{2}(x, t)\right|\left(\frac{\beta(t)-\alpha(t)}{2}\right) d t \\
= & \frac{1}{2}\left[\int_{a+\lambda \frac{\mathrm{b}-\mathrm{a}}{2}}^{x}\left\{t-\left(a+\lambda \frac{b-a}{2}\right)\right\}\{\beta(t)-\alpha(t)\} d t\right. \\
& +\int_{x}^{b-\lambda \frac{\mathrm{b}-\mathrm{a}}{2}}\left\{\left(b-\lambda \frac{b-a}{2}\right)-t\right\}\{\beta(t)-\alpha(t)\} d t .
\end{aligned}
$$

which completes the proof.

Theorem 3.2. Let $f: I \rightarrow R$ be a differentiable function in the interior $I^{0}$ of an interval $I$ in $R$ and $a, b \in I^{0}$ with $a<b$. If $\alpha(x) \leq f^{\prime}(x)$ for any $\alpha \in C\left[a+\lambda \frac{b-a}{2}, b-\lambda \frac{b-a}{2}\right]$ and $x \in\left[a+\lambda \frac{b-a}{2}, b-\lambda \frac{b-a}{2}\right]$ for $\lambda \in[0,1]$, then the following inequalities hold:

$$
\begin{aligned}
& {\left[\int_{a+\lambda \frac{\mathrm{b}-\mathrm{a}}{2}}^{x}\left\{t-\left(a+\lambda \frac{b-a}{2}\right)\right\} \alpha(t) d t\right.} \\
& \left.\quad+\int_{x}^{b-\lambda \frac{\mathrm{b}-\mathrm{a}}{2}}\left\{t-\left(b-\lambda \frac{b-a}{2}\right)\right\} \alpha(t) d t\right] \\
& \quad-\max \left\{x-\left(a+\lambda \frac{b-a}{2}\right),\left(b-\lambda \frac{b-a}{2}\right)-x\right\} \\
& \quad \times\left\{f\left(b-\lambda \frac{b-a}{2}\right)-f\left(a+\lambda \frac{b-a}{2}\right)-\int_{a+\lambda \frac{\mathrm{b}-\mathrm{a}}{2}}^{b-\lambda \frac{\mathrm{b}-\mathrm{a}}{2}} f(t) d t\right\} \\
& \leq \\
& \quad(1-\lambda)(b-a) f(x)-\int_{a+\lambda \frac{\mathrm{b}-\mathrm{a}}{2}}^{b-\lambda \frac{\mathrm{b}-\mathrm{a}}{2}} f(t) d t \\
& \leq \\
& \quad\left[\int_{a+\lambda \frac{\mathrm{b}-\mathrm{a}}{2}}^{x}\left\{t-\left(a+\lambda \frac{b-a}{2}\right)\right\} \beta(t) d t\right. \\
& \left.\quad+\int_{x}^{b-\lambda \frac{\mathrm{b}-\mathrm{a}}{2}}\left\{t-\left(b-\lambda \frac{b-a}{2}\right)\right\} \alpha(t) d t\right] \\
& \quad+\max \left\{x-\left(a+\lambda \frac{b-a}{2}\right),\left(b-\lambda \frac{b-a}{2}\right)-x\right\} \\
& \quad \times\left\{f\left(b-\lambda \frac{b-a}{2}\right)-f\left(a+\lambda \frac{b-a}{2}\right)-\int_{a+\lambda \frac{\mathrm{b}-\mathrm{a}}{2}}^{b-\lambda \frac{\mathrm{b}-\mathrm{a}}{2}} f(t) d t\right\} .
\end{aligned}
$$


Proof. By the definition $K_{2}(\cdot, \cdot)$, we have

$$
\begin{aligned}
& \int_{a+\lambda \frac{\mathrm{b}-\mathrm{a}}{2}}^{b-\lambda \frac{\mathrm{b}-\mathrm{a}}{2}} K_{2}(x, t)\left(f^{\prime}(t)-\alpha(t)\right) d t \\
& =(1-\lambda)(b-a) f(x)-\left[\int_{a+\lambda \frac{\mathrm{b}-\mathrm{a}}{2}}^{x}\left\{t-\left(a+\lambda \frac{b-a}{2}\right)\right\} \alpha(t) d t\right. \\
& \left.+\int_{x}^{b-\lambda \frac{\mathrm{b}-\mathrm{a}}{2}}\left\{t-\left(b-\lambda \frac{b-a}{2}\right)\right\} \alpha(t) d t\right]-\int_{a+\lambda \frac{\mathrm{b}-\mathrm{a}}{2}}^{b-\lambda \frac{\mathrm{b}-\mathrm{a}}{2}} f(t) d t,
\end{aligned}
$$

we have

$$
\begin{aligned}
\mid & (1-\lambda)(b-a) f(x)-\left[\int_{a+\lambda \frac{\mathrm{b}-\mathrm{a}}{2}}^{x}\left\{t-\left(a+\lambda \frac{b-a}{2}\right)\right\} \alpha(t) d t\right. \\
& \left.+\int_{x}^{b-\lambda \frac{\mathrm{b}-\mathrm{a}}{2}}\left\{t-\left(b-\lambda \frac{b-a}{2}\right)\right\} \alpha(t) d t\right]-\int_{a+\lambda \frac{\mathrm{b}-\mathrm{a}}{2}}^{b-\lambda \frac{\mathrm{b}-\mathrm{a}}{2}} f(t) d t \mid \\
\leq & \int_{a+\lambda \frac{\mathrm{b}-\mathrm{a}}{2}}^{b-\lambda \frac{\mathrm{b}-\mathrm{a}}{2}}\left|K_{2}(x, t)\right|\left|f^{\prime}(t)-\alpha(t)\right| d t \\
= & \int_{a+\lambda \frac{\mathrm{b}-\mathrm{a}}{2}}^{b-\lambda \frac{\mathrm{b}-\mathrm{a}}{2}}\left|K_{2}(x, t)\right|\left(f^{\prime}(t)-\alpha(t)\right) d t \\
\leq & t \in\left[a+\lambda \frac{\mathrm{b}-\mathrm{a}}{2}, b-\lambda \frac{\mathrm{b}-\mathrm{a}}{2}\right]\left|K_{2}(x, t)\right| \int_{a+\lambda \frac{\mathrm{b}-\mathrm{a}}{2}}^{b-\lambda \frac{\mathrm{b}-\mathrm{a}}{2}}\left(f^{\prime}(t)-\alpha(t)\right) d t \\
= & \max \left\{x-\left(a+\lambda \frac{b-a}{2}\right),\left(b-\lambda \frac{b-a}{2}\right)-x\right\} \\
& \times\left\{f\left(b-\lambda \frac{b-a}{2}\right)-f\left(a+\lambda \frac{b-a}{2}\right)-\int_{a+\lambda \frac{\mathrm{b}-\mathrm{a}}{2}}^{b} \alpha(t) d t\right\},
\end{aligned}
$$

which completes the proof.

Theorem 3.3. Let $f: I \rightarrow R$ be a differentiable function in the interior $I^{0}$ of an interval $I$ in $R$ and $a, b \in I^{0}$ with $a<b$. If $f^{\prime}(x) \leq \beta(x)$ for any $\beta \in C\left[a+\lambda \frac{b-a}{2}, b-\lambda \frac{b-a}{2}\right]$ and $x \in\left[a+\lambda \frac{b-a}{2}, b-\lambda \frac{b-a}{2}\right]$ for $\lambda \in[0,1]$, then the following inequalities hold:

$$
\begin{aligned}
& {\left[\int_{a+\lambda \frac{\mathrm{b}-\mathrm{a}}{2}}^{x}\left\{t-\left(a+\lambda \frac{b-a}{2}\right)\right\} \beta(t) d t\right.} \\
& \left.\quad+\int_{x}^{b-\lambda \frac{\mathrm{b}-\mathrm{a}}{2}}\left\{t-\left(b-\lambda \frac{b-a}{2}\right)\right\} \beta(t) d t\right]
\end{aligned}
$$




$$
\begin{aligned}
- & \max \left\{x-\left(a+\lambda \frac{b-a}{2}\right),\left(b-\lambda \frac{b-a}{2}\right)-x\right\} \\
& \times\left\{f\left(a+\lambda \frac{b-a}{2}\right)-f\left(b-\lambda \frac{b-a}{2}\right)+\int_{a+\lambda \frac{\mathrm{b}-\mathrm{a}}{2}}^{b-\lambda \frac{\mathrm{b}-\mathrm{a}}{2}} \beta(t) d t\right\} \\
\leq & (1-\lambda)(b-a) f(x)-\int_{a+\lambda \frac{\mathrm{b}-\mathrm{a}}{2}}^{b-\lambda \frac{\mathrm{b}-\mathrm{a}}{2}} f(t) d t \\
\leq & {\left[\int_{a+\lambda \frac{\mathrm{b}-\mathrm{a}}{2}}^{x}\left\{t-\left(a+\lambda \frac{b-a}{2}\right)\right\} \beta(t) d t\right.} \\
+ & \left.\int_{x}^{b-\lambda \frac{\mathrm{b}-\mathrm{a}}{2}}\left\{t-\left(b-\lambda \frac{b-a}{2}\right)\right\} \beta(t) d t\right] \\
+ & \max \left\{x-\left(a+\lambda \frac{b-a}{2}\right),\left(b-\lambda \frac{b-a}{2}\right)-x\right\} \\
& \times\left\{f\left(a+\lambda \frac{b-a}{2}\right)-f\left(b-\lambda \frac{b-a}{2}\right)+\int_{a+\lambda \frac{\mathrm{b}-\mathrm{a}}{2}}^{b-\lambda \frac{\mathrm{b}-\mathrm{a}}{2}} \beta(t) d t\right\} .
\end{aligned}
$$

Proof. By the definition $K_{2}(\cdot, \cdot)$, we have

$$
\begin{aligned}
& \int_{a+\lambda \frac{\mathrm{b}-\mathrm{a}}{2}}^{b-\lambda \frac{\mathrm{b}-\mathrm{a}}{2}} K_{2}(x, t)\left(f^{\prime}(t)-\beta(t)\right) d t \\
& =(1-\lambda)(b-a) f(x)-\int_{a+\lambda \frac{\mathrm{b}-\mathrm{a}}{2}}^{b-\lambda \frac{\mathrm{b}-\mathrm{a}}{2}} f(t) d t \\
& -\left[\int_{a+\lambda \frac{\mathrm{b}-\mathrm{a}}{2}}^{x}\left\{t-\left(a+\lambda \frac{b-a}{2}\right)\right\} \beta(t) d t\right. \\
& \left.\quad+\int_{x}^{b-\lambda \frac{\mathrm{b}-\mathrm{a}}{2}}\left\{t-\left(b-\lambda \frac{b-a}{2}\right)\right\} \beta(t) d t\right] .
\end{aligned}
$$

So, we have

$$
\begin{aligned}
& \mid(1-\lambda)(b-a) f(x)-\int_{a+\lambda \frac{\mathrm{b}-\mathrm{a}}{2}}^{b-\lambda \frac{\mathrm{b}-\mathrm{a}}{2}} f(t) d t \\
& -\left[\int_{a+\lambda \frac{\mathrm{b}-\mathrm{a}}{2}}^{x}\left\{t-\left(a+\lambda \frac{b-a}{2}\right)\right\} \beta(t) d t\right. \\
& \left.\quad+\int_{x}^{b-\lambda \frac{\mathrm{b}-\mathrm{a}}{2}}\left\{t-\left(b-\lambda \frac{b-a}{2}\right)\right\} \beta(t) d t\right] \mid
\end{aligned}
$$




$$
\begin{aligned}
\leq & \int_{a+\lambda \frac{\mathrm{b}-\mathrm{a}}{2}}^{b-\lambda \frac{\mathrm{b}-\mathrm{a}}{2}}\left|K_{2}(x, t)\right|\left|\beta(t)-f^{\prime}(t)\right| d t \\
\leq & \max _{t \in\left[a+\lambda \frac{\mathrm{b}-\mathrm{a}}{2}, b-\lambda \frac{\mathrm{b}-\mathrm{a}}{2}\right]}\left|K_{2}(x, t)\right| \int_{a+\lambda \frac{\mathrm{b}-\mathrm{a}}{2}}^{b-\lambda \frac{\mathrm{b}-\mathrm{a}}{2}}\left(\beta(t)-f^{\prime}(t)\right) d t \\
= & \max \left\{x-\left(a+\lambda \frac{b-a}{2}\right),\left(b-\lambda \frac{b-a}{2}\right)-x\right\} \\
& \times\left\{f\left(a+\lambda \frac{b-a}{2}\right)-f\left(b-\lambda \frac{b-a}{2}\right)+\int_{a+\lambda \frac{\mathrm{b}-\mathrm{a}}{2}}^{b-\lambda \frac{\mathrm{b}-\mathrm{a}}{2}} \beta(t) d t\right\},
\end{aligned}
$$

which completes the proof.

\section{References}

[1] S. S. Dragomir, Some Ostrowski type inequalities for $n$-times differentiable mappings and applications, RGMIA, Research Report Collection, $\mathbf{1}(\mathbf{1})(1998)$.

[2] S. S. Dragomir, Th. M. Rassias(Eds.), Ostrowski type inequalities and applications in numerical integration, Kluwer Academic Publisher (2002).

[3] S. S. Dragomir, S. Wang, An inequality of Ostrowski-Grüss type and its applications to the estimation of error bounds for some special means and for some numerical quadrature rules, Comput. and Math. with Appl.,33(11)(1997) 15-20. 10.1016/s0898-1221(97)00084-9.

[4] Q. Feng, F. Meng, Some generalized Ostrowski-Grüss type integral inequalities, Comput. and Math. with Appl.,63(3)(2012) 652-659. 10.1016/j.camwa.2011.11.017.

[5] M. Matić, J. E. Pečarić, N. Ujević, An inequality of Ostrowski type via Pompeiu's mean value theorem, J. of Inequal. in Pure and Appl. Math., 6(3) (2005) Art No:83.

[6] M. Masjed-Jamei, S. S. Dragomir, An analogue of the Ostrowski inequality and applications, http://ajmaa.org/RGMIA/papers/v14, 14(2011). 
[7] M. Masjed-Jamei, S. S. Dragomir, A new generalization of the Ostrowski inequality and applications, Filomat, 25(1) (2011) 115-123. 10.2298/FIL1101115M.

[8] G. V. Milovanović, J. E. Pečarić, On generalization of the inequality of A. Ostrowski and some related applications, Univ. Beograd Publ. Electrotehn Fak. Ser. Mat. Fiz., 544-576 (1976) 155-158.

[9] D. S. Mitronović, J. E. Pečarić, A. M. Fink, Inequalities involving functions and their integrals and derivatives, Kluwer Academic, Dordrecht (1991).

[10] A. Ostrowski, Über die absolutabweichung einer differentierbaren funktion von ihren integralmittelwert, Comment. Math. Helv., 10(1938) 226-227.

[11] M. E. Özdemir, A. O. Akdemir, Erhan Set, On the Ostrowski-Grüss type inequality for twice differentiable functions, Hacettepe J. of Math. and Stat., 41(5)(2012) 651-655.

[12] C. E. M. Pearce, J. E. Pečarić, N. Ujević, S. Varosanec, Generalizations of some inequalities of Ostrowski-Grüss type, Math. Inequal. Appl., 3(1)(2000) 25-34. 10.7153/mia-03-03.

[13] N. Ujević, New bounds for the first inequality of Ostrowski-Grüss type and applications, Comput. and Math. with Appl., 46(2003) 421-427. 10.1016/s0898-1221(03)90031-6.

[14] S. Yang, A unified approach to some inequalities of OstrowskiGrüss type, Comput. and Math. with Appl., 51(2006) 1047-1056. 10.1016/j.camwa.2005.09.005. 
